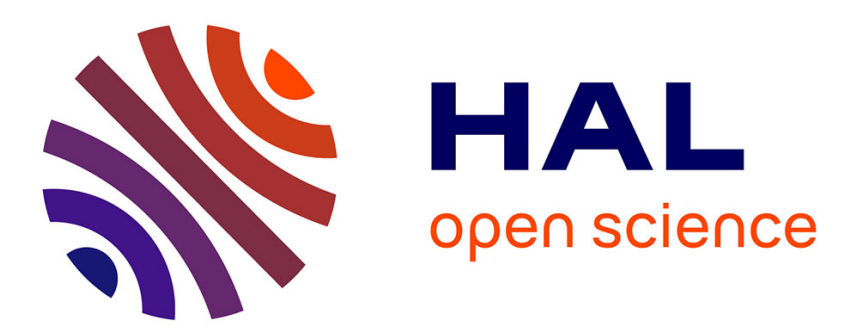

\title{
Adaptive Estimation of Vehicle Dynamics Through RLS and Kalman Filter Approaches
}

Kun Jiang, Alessandro Correa Victorino, Ali Charara

\section{To cite this version:}

Kun Jiang, Alessandro Correa Victorino, Ali Charara. Adaptive Estimation of Vehicle Dynamics Through RLS and Kalman Filter Approaches. 18th IEEE International Conference on Intelligent Transportation Systems (ITSC 2015), Sep 2015, Canary Islands, Spain. pp.1741-1746, 10.1109/ITSC.2015.283 . hal-01243207

\section{HAL Id: hal-01243207 \\ https://hal.science/hal-01243207}

Submitted on 14 Dec 2015

HAL is a multi-disciplinary open access archive for the deposit and dissemination of scientific research documents, whether they are published or not. The documents may come from teaching and research institutions in France or abroad, or from public or private research centers.
L'archive ouverte pluridisciplinaire HAL, est destinée au dépôt et à la diffusion de documents scientifiques de niveau recherche, publiés ou non, émanant des établissements d'enseignement et de recherche français ou étrangers, des laboratoires publics ou privés. 


\title{
Adaptive estimation of vehicle dynamics through RLS and Kalman filter approaches
}

\author{
Kun Jiang, Alessandro Corrêa Victorino, Ali Charara
}

\begin{abstract}
This article presents a new methodology for estimation of vehicle's vertical forces in order to enhance road safety. Direct measurement of vertical forces requires a complex and expensive experimental set-up, which is not acceptable for ordinary passenger cars. The main contribution of this article is providing a reliable estimator of vertical tire forces by using currently available low-cost sensors. The first advantage of the proposed method is that we modified the vehicle model to take into account the roll and pitch dynamics, which makes our estimator stay robust during sharp turning or at inclined road. The other advantage is that we proposed a process to identify the vehicle parameters, instead of regarding them as known constants. This could enable our estimator to stay reliable even when the parameters are wrongly configured. The parameter identification process is based on recursive least squares (RLS) algorithm. The state observers are based on Kalman filter. The estimation process is applied and compared to real experimental data obtained in real conditions. Experimental results validate and prove the feasibility of this approach.
\end{abstract}

\section{INTRODUCTION}

Nowadays many driver safety assistance and stability control systems, such as lane departure warning system (LDWS), adaptive cruise control (ACC), electronic stability program (ESP), are installed on modern vehicles, in order to prevent dangerous situations. A robust estimation of vehicle dynamics states and parameters (such as roll angle and wheel ground forces) is quite essential in this area.

The wheel ground forces, also called as vertical load, have a strong interaction with lateral and longitudinal dynamics[2][4]. A dramatic variation in the vertical load will probably cause a rollover situation, and will also cause a shift in tire's lateral and longitudinal forces[12]. Monitoring the vertical load at each tire in real time is meaningful for vehicle's security. Due to technical and economic reasons, a force sensor that measures directly vertical load is not available in a standard vehicle and is difficult to be installed. Therefore, the motivation of our research is to develop a virtual sensor which uses low-cost sensors and provides reliable estimation of vertical loads.

The difficulties in estimation of vertical forces relies in two aspects, modeling and parameter configuration. In [8], the author presented a 14 DOF (Degree Of Freedom) vehicle model where the roll dynamics are used to calculate vertical tire forces. The Full-car model introduced too many parameters to be configured. In the work of [5][6], a simplified suspension

The authors are with Laboratoire Heudiasyc, CNRS UMR 7253, Sorbonne universités, Université de Technologie de Compiègne, France, funded in part by the Region Picardie VERVE project, MS2T LABEX and ROBOTEX. kun.jiang@hds.utc.fr, acorreau@hds.utc.fr, acharara@hds.utc.fr model is proposed to consider the roll dynamics. However, the vehicle parameters such as weight, roll stiffness are given as a known constant. In [6], an EKF (Extended Kalman Filter) estimator is developed to adapt to the non-linear model. In [8], the DEKF (Dual Extended Kalman Filter) is introduced for estimating lateral forces. During the process, the variation of tire cornering stiffness is also estimated in real time.

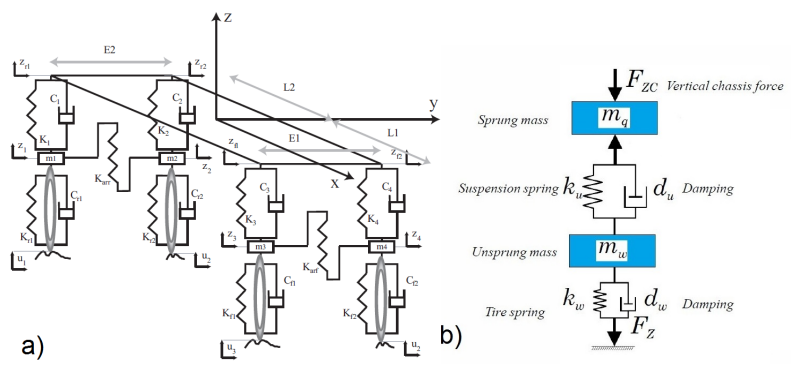

Figure 1. a) Full-car automotive suspension model; b) Quarter-car automotive suspension model

The main contribution of this study consists of two parts. We proposed a new non-linear suspension model to calculate the vertical load at each tire. And we developed an adaptive estimation algorithm based on Recursive Least Square (RLS) algorithm and Kalman Filter (KF) to estimate the vehicle states and configure the vehicle parameters at the same time. The advantage of this method is to enable an accurate estimation of vertical load in presence of different road angles and unknown vehicle parameters.

To evaluate the performance of our observers, the simulation software PROSPER/CALLAS is used. The estimator is also tested with the data acquired from the experimental vehicle DYNA, a Peugeot 308 [5].

Section 2 presents the vehicle models. Section 3 describes the structure of the observer. Then, experiment is described in Section 4. Finally, concluding remarks and future perspectives are given in Section 5.

\section{VEHICLE MODELING}

When pitch and roll dynamics are considered as negligible, the vehicle can be regarded as a rigid body, then the lateral load transfer can be calculated by a very simple model, represented by Equation (1).

$$
\Delta F z_{\text {lat }}=\frac{m_{v} a_{y m} h}{E}
$$

where $m_{v}$ is the total vehicle mass, $a_{y m}$ is the measured lateral acceleration, $E$ is the vehicle width, $h$ is the height of COG (center of gravity), lateral load transfer is defined as $\Delta F z_{l a t}=F_{z 11}-F_{z 12}+F_{z 21}-F_{z 22}$, as a corresponding item, 
the longitudinal transfer of load is defined as $\Delta F z_{l o n}=F_{z 11}-$ $F_{z 12}+F_{z 21}-F_{z 22}$, where $F_{z i j}$ represents the vertical load at each tire, $F_{z 11}$ corresponds to the front left tire.

However, in real situation, such as in sharp turning, the pitch and roll dynamics will cause a significant shift in load transfer. To compensate these errors, we proposed to consider suspension system in vehicle model.

\section{A. Vehicle model with suspension system}

Suspension systems affect the handling and the ride quality of cars, so they are currently of great interest to both academia and industry. A full car suspension model is introduced in [10].

The full car suspension model shown in Figure 1-a) has 7 Degree-Of-Freedom (DOF) and lots of parameters, which is too complicated to be used as state update model in a Kalman filter. In order to represent the vehicle vertical dynamics more clearly, a simplified 2 DOF quarter-car model is proposed in [14], shown in Figure 1-b).

The motion of suspension system represents vehicle's roll and pitch dynamics. Based on the geometry of the roll motion, we can estimate the roll angle $\phi_{v}$ and pitch angle $\theta_{v}$ with the suspension defections:

$$
\phi_{v}=\frac{\sigma_{11}-\sigma_{12}+\sigma_{21}-\sigma_{22}}{2 E}, \theta_{v}=\frac{\sigma_{11}+\sigma_{12}-\sigma_{21}-\sigma_{22}}{2\left(L_{1}+L_{2}\right)}
$$

where $\sigma_{i j}$ is the relative motion of the body with respect to the wheels, $L_{1}$ is the distance between COG and front axle, $L_{2}$ is the distance between COG and rear axle

The location of roll and pitch axis could vary during the driving. To simplify the model, we assume in the top view both roll and pitch axis pass the COG. Supposing all the vehicle mass is sprung, then the lateral load transfer could be formulated as:

$$
\Delta F z_{l a t}=\frac{K_{\phi} * \phi_{v}}{E}
$$

where $K_{\phi}$ is the equivalent roll stiffness.

However, in a real vehicle, neither the sprung mass (vehicle body) nor the non-sprung mass (wheels, axles) could be ignored. As a result, the vehicle model we proposed is the weighted sum of the two models in Equation (1) and (3). Then the lateral load transfer can be calculated by Equation (4)

$$
\Delta F z_{\text {lat }}=\left(\alpha * m_{v} a_{y m} h+(1-\alpha) * K_{\phi} \phi_{v}\right) \frac{1}{E}
$$

where $\alpha$ is the percentage of the torque caused by nonsprung mass.

Similar to the lateral load transfer, the longitudinal load transfer is also affected by both sprung mass and unsprung mass, and it could be calculated by Equation (5).

$$
\Delta F z_{l o n}=m\left(\frac{L_{2}-L_{1}}{L}\right) a_{z m}+\frac{\left(\alpha \cdot m_{v} a_{x m} h+(1-\alpha) \cdot K_{\theta} \theta\right)}{L}
$$

where $a_{x m}, a_{y m}, a_{z m}$ is the measured acceleration in each direction.

The proposed model is illustrated in Figure 2. The advantage is to take into account the roll and pitch dynamics.

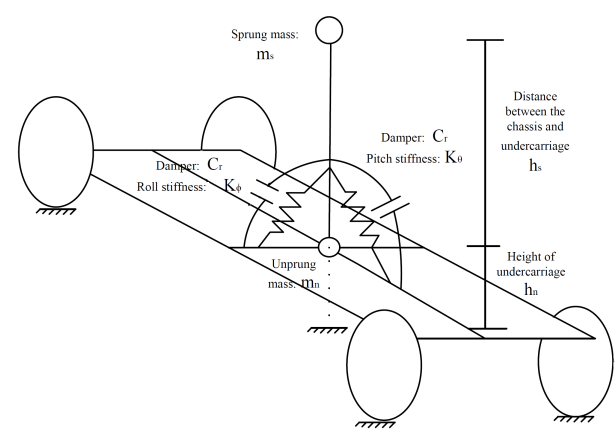

Figure 2. Automotive suspension model considering pitch and roll dynamics

\section{B. Identification of vehicle parameters}

This section describes the mathematical models we used to identify the value of vehicle parameters. In real driving condition, the vehicle parameters can vary a lot, such as the weight of a empty vehicle or a full loaded vehicle. For this reason, we proposed to regard these parameters as unknown and to be estimated. The parameters we will configure in this article, shown in Table 1 , are vehicle mass, roll stiffness of suspension system, road angles and position of the COG, which in this paper means $L_{1}$.

Table I

PARAMETERS OF THE VEHICLE

\begin{tabular}{|c|c|c|c|}
\hline & parameters & & parameters \\
\hline \hline$m_{v}$ & whole vehicle weight & $K_{\phi}$ & roll stiffness \\
\hline$\phi_{r}$ & bank angle of the road & $\theta_{r}$ & incline angle of the road \\
\hline$L_{1}$ & position of COG & $K_{\theta}$ & pitch stiffness \\
\hline
\end{tabular}

1) Vehicle mass: Nowadays, many controlled suspensions are equipped with relative position sensors to measure suspension deflections $\sigma_{i j}$ at each corner. At a passive suspension with linear spring characteristics, and by neglecting the wheel deflection, a variation of sprung mass at each corner $\triangle m_{s i j}$ changes the spring deflection $\sigma_{i j} \rightarrow \sigma_{i j}+\triangle_{i j}$ where

$$
\triangle m_{s i j}=\frac{k_{s} \triangle_{i j}+F_{\text {internal }}}{a_{z}}
$$

where $\triangle_{i j}$ is the spring deflection variation, $F_{\text {internal }}$ is the internal forces between each quarter of the body, which could be introduced by the lateral and longitudinal accelerations. One method to eliminate the term of internal force is to estimate the mass when the vehicle is at rest, as $F_{\text {internal }}=0$ [6]. Another method to eliminate the $F_{\text {internal }}$ is to calculate the total mass of the vehicle, because the sum of internal forces will be zero.

$$
m_{v}=m_{e}+\sum \triangle m_{s i j}=m_{e}+\frac{k_{s}}{a_{z}} \sum \triangle_{i j}
$$

where $m_{e}$ is the mass of empty vehicle,

2) Roll and pitch stiffness: The roll and pitch stiffness is the key parameter to describe the roll and pitch dynamics of vehicle, as shown in Equation (8).

$$
\begin{aligned}
I_{x x} \ddot{\phi}_{v} & =-C_{d a m} \dot{\phi}_{v}-K_{\phi} \phi_{v}+m_{v} h a_{y m} \\
I_{y y} \ddot{\theta}_{v} & =-C_{d a m} \dot{\theta}_{v}-K_{\theta} \theta_{v}+m_{v} h a_{x m}
\end{aligned}
$$

Where $I_{x x}$ is the moment of inertia of the vehicle with respect to the roll axis, $I_{y y}$ is respect to the pitch axis and $h_{s}$ 
is the height of the sprung mass about the roll axis, $C_{d a m}$ is the suspension damping coefficient.

In a dynamic driving situation, the relationship between $a_{y m}$ and $\phi_{v}$ is expressed by a transfer function:

$$
\phi_{v}(s)=\frac{m_{v} h}{I_{x x} s^{2}+C_{\phi} s+K_{\phi}} a_{y m}(s)
$$

As we can see in Equation (9), the roll angle is a retarded response to the lateral acceleration, which explains the phase lag between $a_{y m}$ and $\phi_{v}$.

However, in an approximate static driving situation, we assume $\ddot{\phi}_{v}=\dot{\phi}_{v}=0$, then we have $K_{\phi} \phi_{v}=m_{v} h_{s} a_{y m}$. The roll stiffness could be configured by:

$$
K_{\phi}=\frac{m_{v} h a_{y m}}{\phi_{v}}
$$

3) The position of $C O G$ : The pitch moment balance equation with respect to pitch axis is expressed by Equation (11).

$$
F z_{f} * L_{1}-F z_{r} * L_{2}=-m_{v} h_{s} a_{x m}
$$

where the vertical load at front axle is defined as $F z_{f}=$ $F z_{11}+F z_{12}$, vertical load at rear axle is $F z_{r}=F z_{21}+F z_{22}$.

As we can see in (11), the position of gravity center has a direct impact on the distribution of load. However, in the literature the position of COG is seldom discussed and normally supposed be a known constant. In this article we proposed a novel method to identify this parameter $\left(L_{1}\right)$. Our method is analyzing vehicle's lateral dynamics. The position of COG not only affects the vertical load, but also influences the distribution of lateral forces. For design simplicity, the singletrack vehicle model (also called the bicycle model) is usually used in estimator design, shown in Figure 3.

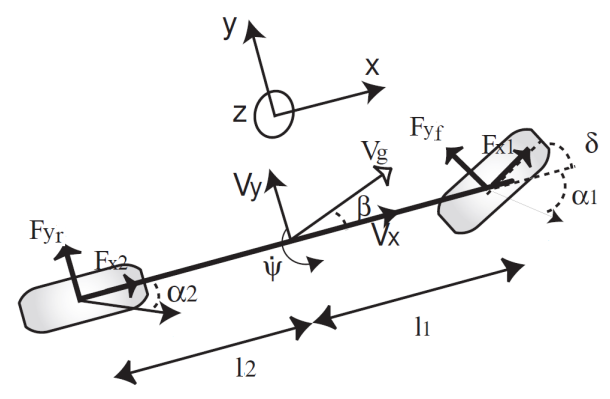

Figure 3. Bicycle model

The yaw moment balance equation with respect to $\mathrm{COG}$ is expressed by Equation (12).

$$
I_{z} \dot{\omega}=L_{1}\left(F x_{f} \sin \delta_{f}+F y_{f} \cos \delta_{f}\right)-L_{2} F y_{r}+M_{z}
$$

where $\delta_{f}$ is steering angle, $I_{z}$ is the inertial moment with respect to yaw axis, external yaw moment $M_{z}$ exits when the braking forces of left and right wheels are different,

By assuming that $\delta_{f}$ is relatively small, the lateral and yaw rate dynamics, are obtained as follows:

$$
I_{z} \dot{\omega}=L_{1} F y_{f}-L_{2} F y_{r}
$$

where lateral forces at front axle is defined as $F y_{f}=F y_{11}+$ $F y_{12}$, that at rear axle is $F y_{r}=F y_{21}+F y_{22}, L$ is the distance between two axles, $L=L_{1}+L_{2}$. Then we have

$$
L_{1}=\frac{I_{z} \dot{\omega}+L F y_{r}}{F y_{f}+F y_{r}}
$$

With Equation (14), the problem of finding COG position transfers to the estimation of lateral forces.

For small tire slip angles, the lateral tire forces can be linearly approximated as follows:

$$
\begin{gathered}
F y_{f}=-2 C_{f}\left(\beta+\omega L_{1} / v_{x}-\delta_{f}\right) \\
F y_{r}=-2 C_{r}\left(\beta-\omega L_{2} / v_{x}\right) \\
F y_{f}+F y_{r}=m_{v} a_{y m}
\end{gathered}
$$

where $\beta$ is vehicle's slip angle, $C_{f}$ and $C_{r}$ are the cornering stiffness at front and rear wheels.

The vehicle's slip angle $\beta$ is not discussed in this paper. To eliminate the $\beta$ in (15), we assume the cornering stiffness of front and rear wheels are identical, $C_{f}=C_{r}$. With the Equation (14) and (15), we can get our model for the estimation of vehicle's COG as shown in Equation (16).

$$
L_{1}=\frac{I_{z} \dot{\omega}+L\left(\frac{1}{2} m_{v} a_{y m}+C_{f} \omega L / v_{x}-C_{f} \delta_{f}\right)}{m_{v} a_{y m}}
$$

4) Road angles: The measured accelerations contain the information of road angle. With Equation (17) we could estimate the road angles $\phi_{r}, \theta_{r}$. During normal driving situation, the lateral speed is very small compared to longitudinal speed. To simplify our process of parameter identification, we estimate the road angles only at static driving situation, then we can assume the $v_{y}=\dot{v}_{y} \simeq 0$.

$$
\begin{aligned}
& a_{x m}=\dot{v}_{x}-v_{y} \dot{\psi}+\theta_{r} g+\sigma_{a x} \\
& a_{y m}=\dot{v}_{y}+v_{x} \dot{\psi}+\phi_{r} g+\sigma_{a y}
\end{aligned}
$$

\section{OBSERVER DESIGN}

The overall estimation process of the observer is divided into 3 blocks as shown in Figure 4. The first block identifies the vehicle's mass, roll and pitch stiffness, and position of COG. A recursive least squares (RLS) algorithm is utilized based on the mathematical models presented in Section 2. Then the identified vehicle parameters will be used to configure the following blocks. The aim of the second block is to calculate the longitudinal and lateral load transfer based on the new suspension model we proposed. The estimated values will be considered as an essential measure for the third block, which is to estimate the vertical tire forces at each tire. The observer of vertical dynamics state is based on Kalman Filter. The strategy of using cascaded observers allows us to avoid the observability problems, thus enabling the estimation process to be carried out in a simple and practical way [6]. 


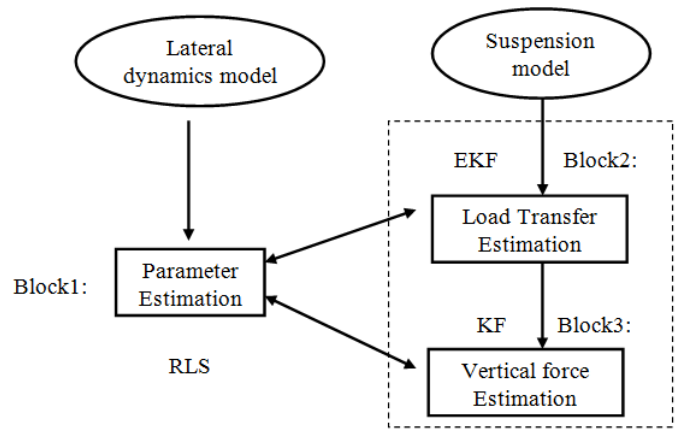

Figure 4. Structure of our observer based on dual extended Kalman filter

\section{A. First block: Parameter Estimation}

The identification of vehicle mass is based on the linear spring characteristics, which could be calculated directly by Equation (7). For other parameters, to minimize the errors caused by different sensor noises, we used the recursive least squares (RLS) algorithm to formulate parameter models, shown in Equation (18). The advantage of using RLS algorithm, is the reduction of the parameter uncertainties without changing the original state observer.

$$
y(t)=\varphi^{T}(t) \theta(t)
$$

where the estimated parameter $\theta(t)$, input regression $\varphi^{T}(t)$, and measured output $y(t)$ are given as

$$
\begin{gathered}
\theta(t)=\left[K_{\phi}, K_{\theta}, L 1, \phi_{r}, \theta_{r}\right]^{T} \\
\varphi^{T}(t)=\operatorname{diag}\left[\phi_{v}, \theta_{v}, m_{v} a_{y m}, g, g\right] \\
y(t)=\left[m_{v} h_{s} a_{y m}, m_{v} h_{s} a_{x m},\right. \\
I_{z} \dot{\omega}+L\left(\frac{1}{2} m_{v} a_{y m}+C_{f} \omega L / v_{x}-C_{f} \delta_{f}\right), \\
\left.a_{x m}-\dot{v}_{x}, a_{y m}-v_{x} \dot{\psi}\right]
\end{gathered}
$$

The recursive process of the RLS algorithm [15], in a Kalman filter interpretation, is described as:

$$
\begin{gathered}
\hat{\theta}(t+1)=\hat{\theta}(t)+K(t+1) \cdot \varepsilon(t+1 \mid \hat{\theta}(t)) \\
\varepsilon(t+1 \mid \hat{\theta}(t))=y(t+1)-\hat{y}(t+1 \mid \hat{\theta}(t)) \\
=y(t+1)-\varphi^{T}(t+1) \cdot \hat{\theta}(t) \\
K(t+1)=P(t) \varphi(t+1)\left[\lambda I+\varphi^{T}(t+1) P(t) \varphi(t+1)\right]^{-1} \\
P(t+1)=\frac{1}{\lambda}\left[I-K(t+1) \varphi^{T}(t+1)\right] P(t)
\end{gathered}
$$

where $I$ is the identity matrix, $\varepsilon(t)$ is the prediction error, and $K(t)$ and $P(t)$ are the Kalman gain and covariance matrices, respectively, $\lambda$ is the forgetting factor. The smaller $\lambda$ is, the less weight is assigned to the older data.

\section{B. Second block: Estimation of load transfer}

An Kalman filter is formulated based on the non-linear vehicle model presented in Section 2, to estimate the load transfer. To build a Kalman Filter, the vertical force system has been represented by a set of discrete state-space equations:

$$
\begin{gathered}
x_{k}=A x_{k-1}+B u_{k-1}+\omega_{k} \\
y_{k}=H x_{k}+v_{k}
\end{gathered}
$$

Where $A$ is the states update matrix, $H$ is the measurement matrix, $\omega_{k}, v_{k}$ are the noises of the models and measurements. The state vector to be estimated is $x_{k}=$ $\left[\phi_{v}, \dot{\phi}_{v}, \theta_{v}, \dot{\theta_{v}}, \triangle F z_{l a t}, \triangle F z_{l o n}, a_{x}, a_{y},\right]$. The state update model is represented by Equations (4) (8). The measurements we used in this block are the accelerations and deflections which are measurable values.

\section{Third block: Estimation of observer parameters}

As the load transfer is estimated in the second block and used as a known measurement in this block. Then the vertical force at each tire can be represented by a very simple linear model represented by Equation (21).

The state vector and measurement matrix are given as:

$$
\begin{aligned}
& X_{1}=\left[\begin{array}{lllll}
F_{z 11} & F_{z 12} & F_{z 21} & F_{z 22}
\end{array}\right]^{T} \\
& \begin{array}{c}
Y_{1}=\left[m_{v} a_{z m}, \frac{1}{2} \Delta F z_{\text {lon }}, \frac{1}{2} \Delta F z_{\text {lon }}, \Delta F z_{\text {lat }} \frac{L_{2}}{L}, \Delta F z_{\text {lat }} \frac{L_{1}}{L}\right]^{T} \\
H_{1}=\left[\begin{array}{cccc}
1 & 1 & 1 & 1 \\
1 & 0 & -1 & 0 \\
0 & 1 & 0 & -1 \\
1 & -1 & 0 & 0 \\
0 & 0 & 1 & -1
\end{array}\right]
\end{array}
\end{aligned}
$$

\section{EXPERIMENTAL VALIDATION}

The algorithm developed here is implemented in MATLAB to realize the off-line estimation. Simulated data generated by vehicle simulation software Callas is used to evaluate our estimator. Our simulation test is conducted at a banked road, the bank angle is set as $30 \%$. Then the data acquired from an experimental car DYNA, shown in Figure 5, is used to test the reliability of our estimator in real conditions.

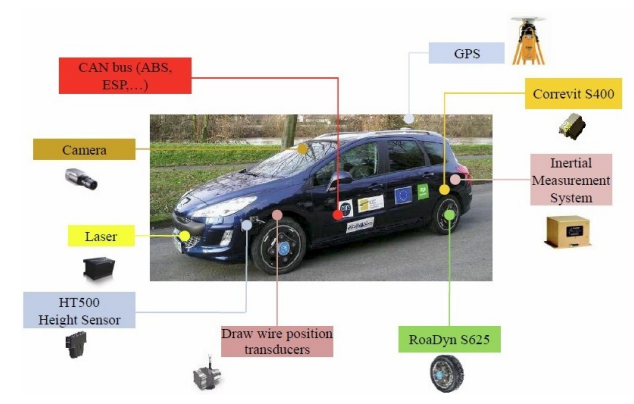

Figure 5. Experimental vehicle DYNA, equipped with different sensors

\section{A. Simulation of Chicane test with wrong configuration of vehicle parameters}

A particular situation of the test is that the vehicle parameters are wrongly configured and should be estimated. Figure 6 presents the final results of our estimated vertical load transfer. In Figure 6, the red lines represent simulation reference data, and the other lines the estimated values. The initial configured value of vehicle mass and roll stiffness is two times bigger than the real value. Without correction of the parameters, the estimation result of load transfer is far from the the reference data, shown by green lines in Figure 6. In contrast, the estimator with parameter estimation block, the observer of vertical load stays reliable, shown by blue lines. 

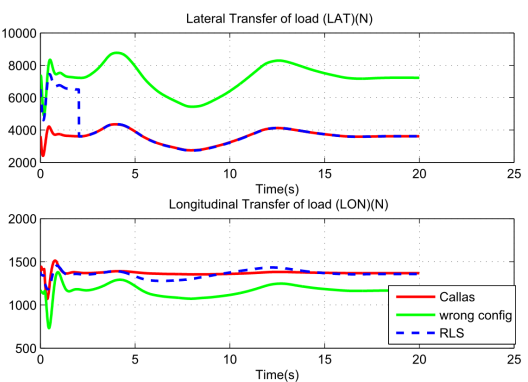

Figure 6. Estimation of load transfer at banked road with wrong configuration "wrong config" represents the estimated values with wrong configuration, "RLS" refers to the proposed auto-configuration estimator.

\section{B. Experiments in real condition}

In order to evaluate our estimator in real driving condition, our experimental car DYNA is arranged to have a sharp turning at inclined road. Figure 7 shows the trajectory, altitude, steering wheel angle and speed during the test.
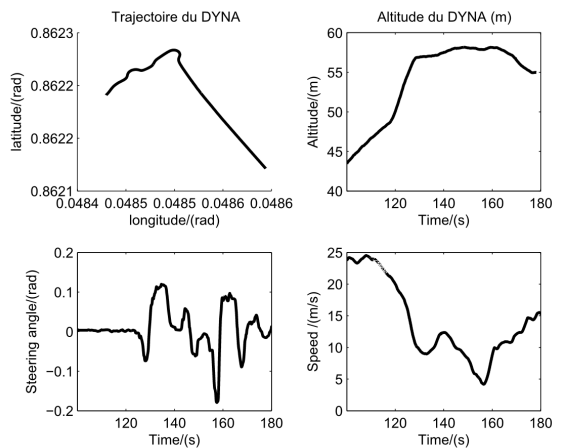

Figure 7. Driving conditions during real car experiment

Experimental results of roll angle obtained from inertial sensors and suspension deflections are compared in Figure 8. The integration method will accumulate considerable errors, as shown by the black line. In contrast, the suspension deflections method shows more accurate results without integration errors.

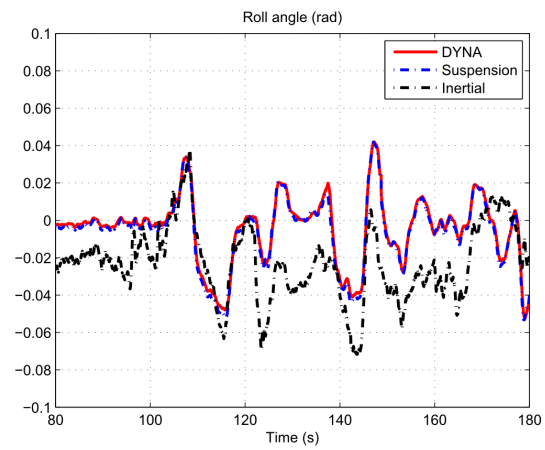

Figure 8. Estimation of roll angle in real conditions. "Suspension" corresponds to the estimated values , "DYNA" refers to the measured data, "Inertial" refers to the integration of roll rate.

The estimated roll and pitch angle will be analyzed in our state model to have a better understanding of driving condition.
With this advantage, the new estimator could provide more accurate and reliable estimation of vertical load. The observed load transfer from the estimators with and without considering roll/pitch angle are compared in Figure 9.
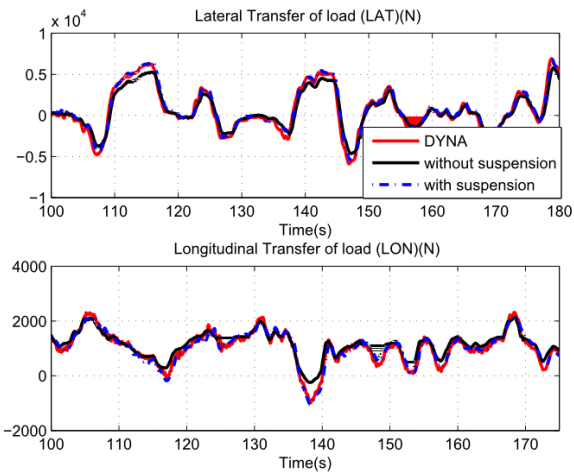

Figure 9. Estimation of load transfer in real conditions. "DYNA" refers to the measured data, "with suspension" corresponds to the estimated values considering roll dynamics, "without suspension" represents the estimation that ignored roll dynamics.

In Figure 9, we can see that in the peak regions, the estimation results with suspension models, represented by blue line, is closer to the reference data. In the peak regions, the vehicle is through a sharp turning which leads to a considerable roll movement. The estimator we proposed could provide a good estimation of load transfer in this situation, as it considered roll dynamics. Then we can continue to estimate the vertical force at each tire, as shown in Figure 10.
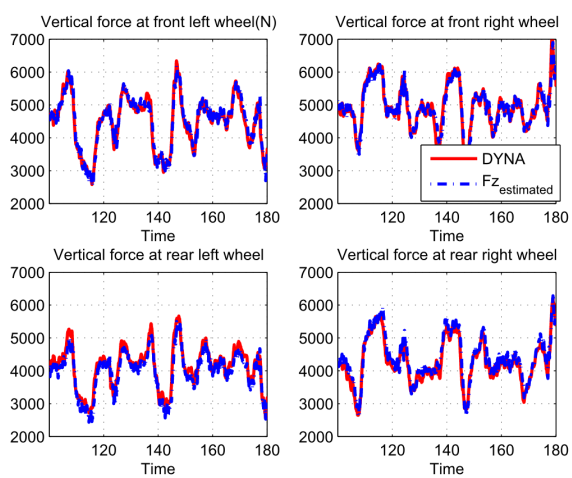

Figure 10. Comparison of estimated tire vertical forces with real data measured by our experimental car. "Estimated" corresponds to the estimated values.

The above experimental results are obtained under the condition that all vehicle parameters are correct. In real condition, the preset parameters can't be accurate. The advantage of our estimator is the estimation of vehicle parameters during driving. Figure 11 shows the comparison between measured value and estimated value of vehicle's total weight in real condition. As explained in Section 2, the deflection of suspension could effectively represent the variation of vehicle's total vertical force and total mass. 


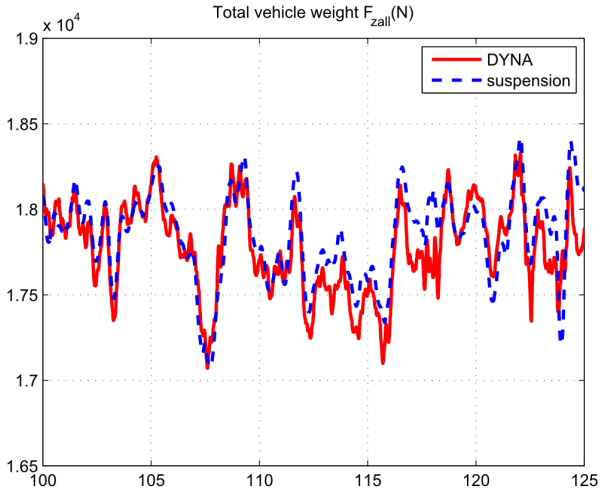

Figure 11. Comparison of estimated total vehicle weight with real data. "suspension" corresponds to the estimated weight by suspension deflection.

Another parameter to be estimated is the position of COG, which influence both the vertical and lateral dynamics. In our process of estimating lateral forces, we didn't use sideslip angle, instead we estimated the lateral force transfer $\left(F y_{f}-F y_{r}\right)$ and total lateral force $\left(F y_{f}+F y_{r}\right)$, shown in Figure 12.
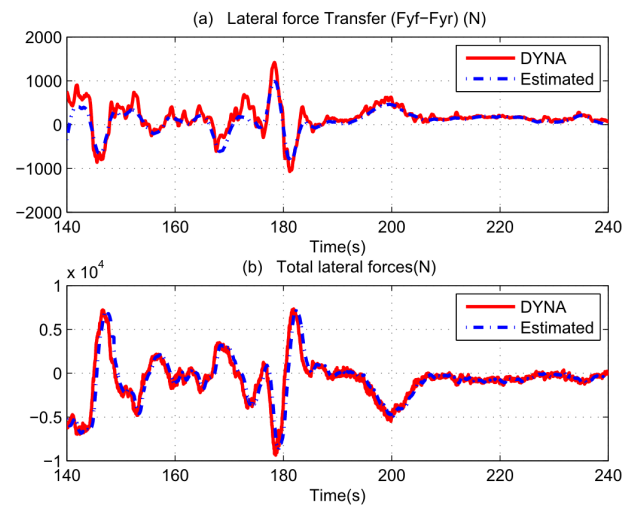

Figure 12. Comparison of estimated lateral forces distribution with directly measured data (DYNA). (a) presents the lateral force transfer $\left(F y_{f}-F y_{r}\right)$, (b) presents the total lateral force.

Then we can estimate the lateral force at front and rear axle respectively. The estimated position of COG is shown in Figure 13.

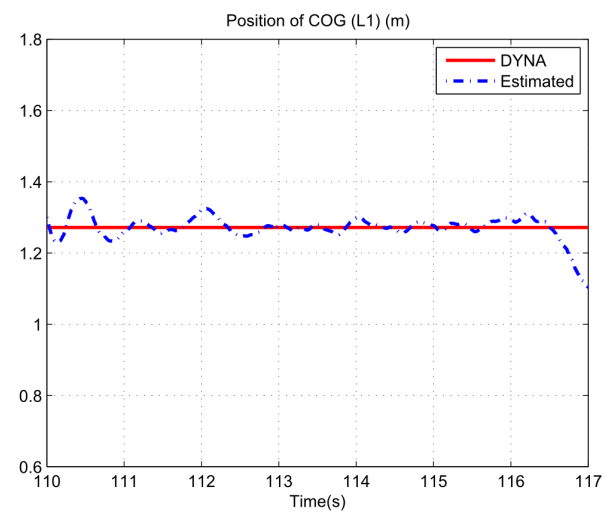

Figure 13. Estimated position of COG
With all the results above, we can confirm that the estimator proposed here could have a good estimation of vehicle parameters. When the parameter is wrongly configured, the parameter estimation block can make the estimator stay accurate and reliable.

\section{CONCLUSIONS AND PROSPECTS}

This paper has presented a new algorithm to estimate vertical road/tire forces. We introduced suspension system into vehicle model to consider the roll and pitch dynamics. We also combined vertical dynamics and lateral dynamics to estimate vehicle parameters. A reliable estimator of vertical dynamics state is developed based on EKF and RLS algorithm. The parameter estimation block makes the observer more robust. Simulated and experimental results are presented to evaluate the performance of the new observer. Several critical tests are performed to compare and validate the new algorithm. The observer gives accurate estimating results even when the vehicle parameters are initially unknown and when the road geometry is varying.

In the future study, we will focus on developing an integrated estimator to observe dynamics states in real time in three directions (vertical, lateral and longitudinal).

\section{REFERENCES}

[1] K.Jiang, A.Pavelescu, A.C.Victorino \& A.Charara. "Estimation of vehicle's vertical and lateral tire forces considering road angle and road irregularity". In 17th IEEE conference of Intelligent Transportation Systems (ITSC), (2014) :342-347.

[2] M.Gadola, D.Chindamo, et al. "Development and validation of a Kalman filter-based model for vehicle slip angle estimation " Vehicle System Dynamics 52.1(2014): 68-84.

[3] Xu Li, Xiang Song, Chingyao Chan. "Reliable vehicle sideslip angle fusion estimation using low-cost sensors ."Measurement 51 (2014): 241 258.

[4] M.Ouahi, J.Stéphant and D.Meiezl. "Simultaneous observation of the wheels' torques and the vehicle dynamic state." Vehcicle System Dynamics 51.5 (2013):737-766.

[5] B. Wang, A. C. Victorino and A. Charara, "State observers applied to vehicle lateral dynamics estimation: a comparison between Extended Kalman filter and Particle filter", 39th Annual Conference of the IEEE Industrial Electronics Society, Austria, Nov, 2013.

[6] D.Moustapha, A.C.Victorino, A. Charara et al. "An estimation process for vehicle wheel-ground contact normal forces." 17th IFAC World Congress, Milano, Italy (2008)

[7] SORNIOTTI, Aldo et D'ALFIO, Nicolò. Vehicle dynamics simulation to develop an active roll control system. SAE Technical Paper, 2007.

[8] Shim, Taehyun, and Chinar Ghike. "Understanding the limitations of different vehicle models for roll dynamics studies." Vehicle system dynamics 45.3 (2007): 191-216.

[9] T.A.Wenzel, K.J.Burnham, M.V.Blundell, R.A.Williams. "Dual extended Kalman filter for vehicle state and parameter estimation" Vehicle System Dynamics 44.2(2006)153-171.

[10] Rajamani, R. (2006). Vehicle dynamics and control, Springer

[11] Ryu, Jihan, and J. Christian Gerdes. "Estimation of vehicle roll and road bank angle." American Control Conference, 2004. Proceedings of the 2004. Vol. 3. IEEE, 2004.

[12] Aleksander, H., Brown, T. \& Martens, J. (2004). Detection of vehicle rollover, Proceedings of the SAE World congress, Michigan, USA.

[13] A. Hac, T. Brown and J. Martens. Detection of vehicle rollover. Vehicle Dynamics \& Simulation, 2004.

[14] D. L. Milliken, E. M. Kasprak, L. Daniel Metz and W. F. Milliken. Race car vehicle dynamics. SAE International, 2003.

[15] Nam, Kanghyun, et al. "Estimation of sideslip and roll angles of electric vehicles using lateral tire force sensors through RLS and Kalman filter approaches." Industrial Electronics, IEEE Transactions on 60.3 (2013): 988-1000 